\title{
Thickness of electrical double layer. Effect of ion size
}

\author{
Klemen Bohinc $^{a}$, Veronika Kralj-Iglič ${ }^{b, *}$, Aleš Iglič ${ }^{a}$ \\ a Faculty of Electrical Engineering, Laboratory of Applied Physics, Tržaška 25, SI-1000 Ljubljana, Slovenia \\ ${ }^{\mathrm{b}}$ Institute of Biophysics, Faculty of Medicine, Lipičeva 2, SI-1000 Ljubljana, Slovenia
}

Received 19 March 2001

\begin{abstract}
The thickness of a single flat electrical double layer is considered. The electrostatic mean field and the excluded volume effect are taken into account. A simple statistical mechanical approach is used, where the particles in the solution are distributed over a lattice with an adjustable lattice constant. Different sizes of ions are described by different values of the lattice constant. Two measures are introduced that describe an effective thickness of the electrical double layer: a distance where the density of the number of counterions drops to a chosen fraction of its maximal value, and a distance defining a region that contains a chosen fraction of the excess of the counterions. It is shown that the effective thickness of the electrical double layer increases with increasing counterion size and with decreasing bulk concentration of ions, whereas increasing the surface charge may cause either a decrease or an increase of the effective thickness. It is shown that the description of the effective thickness of the electrical double layer by the Debye length differs qualitatively from the description presented for low bulk concentrations of ions. (c) 2001 Elsevier Science Ltd. All rights reserved.
\end{abstract}

Keywords: Electrical double layer; Debye length; Electrolyte; Excluded volume effect; Ion condensation

\section{Introduction}

A flat electrical double layer is created by a charged plane in contact with an electrolyte solution composed of solvent molecules, counterions (ions with the charge of the opposite sign than the plane) and coions (ions with the charge of the same sign as the plane). Phenomena involving the electrical double layer are common in systems in contact with the electrolyte solution, such as biological and artificial membranes [1], in liquid crystals [2], in clays [3], and solid electrolytes [4].

As these systems are of importance in technology and medicine, the electrical double layer has been a subject

\footnotetext{
* Corresponding author. Tel.: + 386-1-5437620; fax: + 386$1-4315127$.

E-mail address: vera.kralj-iglic@biofiz.mf.uni-lj.si (V. KraljIglič).
}

of extensive study since the pioneering work of Gouy [5] and Chapman [6] (for reviews see Refs. [1,7]). The Poisson-Boltzmann theory, where dimensionless ions are treated within the mean field approach, is an acknowledged and widely used description.

One of the issues that still needs further clarification, and which this work addresses, is ion-specific behavior [8-12]. Here, we focus on the thickness of the flat electrical double layer, a property that measures the range of the electrostatic influence of the charged plane in the surroundings. We would like to upgrade the description within the Poisson-Boltzmann theory by considering the effect of the size of the ions on the thickness of the electrical double layer. We use a transparent, nearly analytical, but yet detailed enough model, based on the excluded volume effect, that was stated in the 1950s [13-15], and which has recently been developed further [16-20]. 


\section{Theory}

We imagine a single planar surface at $x=0$ with surface charge density $\sigma$. This charged plane of area $A$ is in contact with a solution of symmetric electrolyte, composed of one kind of coions, one kind of counterions and a solvent. For simplicity, we consider a symmetric univalent electrolyte, the generalization being straightforward. The area $A$ is considered to be large, so that boundary effects can be neglected. The solution where $n_{0 \mathrm{~d}}$ is the density of the number of the solvent molecules far from the charged plane, $n_{\mathrm{d}}$ is the density of the number of the counterions and of the coions far from the charged plane, $v_{\mathrm{ct}}$ and $v_{\mathrm{co}}$ are the valences of the counterions and the coions respectively, $v_{\mathrm{ct}}, v_{\mathrm{co}}= \pm$ $1, e_{0}$ is the elementary charge, $\Phi(x)$ is the potential of the mean electrostatic field, $k$ is the Boltzmann constant and $T$ is the temperature.

The mean electrostatic potential is obtained by solving the differential equation

$$
\frac{\mathrm{d}^{2} \Phi(x)}{\mathrm{d} x^{2}}=-\frac{e_{0} n_{\mathrm{s}} n_{\mathrm{d}}\left(v_{\mathrm{ct}} \exp \left(-\frac{v_{\mathrm{ct}} e_{0} \Phi(x)}{k T}\right)+v_{\mathrm{co}} \exp \left(-\frac{v_{\mathrm{co}} e_{0} \Phi(x)}{k T}\right)\right)}{\varepsilon \varepsilon_{0} n_{0 \mathrm{~d}}\left(1+\frac{n_{\mathrm{d}}}{n_{0 \mathrm{~d}}}\left(\exp \left(-\frac{v_{\mathrm{ct}} e_{0} \Phi(x)}{k T}\right)+\exp \left(-\frac{v_{\mathrm{co}} e_{0} \Phi(x)}{k T}\right)\right)\right)}
$$

extends in the positive $x$ direction up to a distance $d$ where the effect of the charged plane is negligible. The system is described by the mean electrostatic field, whereas the finite size of the particles that compose the solution is considered by means of the excluded volume effect. It is assumed that the electrostatic field extends into the positive $x$ direction, whereas there is no electrical field on the other side of the charged plane $(x<0)$. The excluded volume effect is introduced by means of a statistical mechanical description. Within this description each particle in the solution occupies one, and only one, site of a finite volume. A lattice with an adjustable lattice constant is introduced, all sites of this lattice being occupied:

$n_{\mathrm{s}}=n_{\mathrm{ct}}(x)+n_{\mathrm{co}}(x)+n_{\text {solvent }}(x)$

where $n_{\mathrm{ct}}$ is the density of the number of the counterions, $n_{\text {co }}$ is the density of the number of coions and $n_{\text {solvent }}$ is the density of the number of the solvent molecules. The density of the number of lattice sites of the system $n_{\mathrm{s}}$ can be expressed as $n_{\mathrm{s}}=1 / V_{\mathrm{s}}$, where $V_{\mathrm{s}}$ is the volume of a three-dimensional cubic lattice with a lattice side size of $a: V_{\mathrm{s}}=a^{3}$. The volume of the whole system is the sum of the volumes of ions and solvent molecules.

The derivation of the general equations of the model is given elsewhere [18]. The densities of the number of the respective particles depend on the distance from the charged plane

$$
\begin{aligned}
& n_{\mathrm{ct}}(x)=\frac{n_{\mathrm{s}} n_{\mathrm{d}} \exp \left(-\frac{v_{\mathrm{ct}} e_{0} \Phi(x)}{k T}\right)}{n_{0 \mathrm{~d}}\left(1+\frac{n_{\mathrm{d}}}{n_{0 \mathrm{~d}}}\left(\exp \left(-\frac{v_{\mathrm{ct}} e_{0} \Phi(x)}{k T}\right)+\exp \left(-\frac{v_{\mathrm{co}} e_{0} \Phi(x)}{k T}\right)\right)\right)} \\
& n_{\mathrm{co}}(x)=\frac{n_{\mathrm{s}} n_{\mathrm{d}} \exp \left(-\frac{v_{\mathrm{co}} e_{0} \Phi(x)}{k T}\right)}{n_{0 \mathrm{~d}}\left(1+\frac{n_{\mathrm{d}}}{n_{0 \mathrm{~d}}}\left(\exp \left(-\frac{v_{\mathrm{ct}} e_{0} \Phi(x)}{k T}\right)+\exp \left(-\frac{v_{\mathrm{co}} e_{0} \Phi(x)}{k T}\right)\right)\right)}
\end{aligned}
$$

We chose

$\Phi(d)=0$ $d \rightarrow \infty$.

$n_{\mathrm{ct}}\left(x_{1 / 2}\right)-n_{\mathrm{d}}=\frac{1}{2}\left(n_{\mathrm{ct}}(0)-n_{\mathrm{d}}\right)$ $x=0$.

Accordingly, $d$ should be regarded as very large, i.e.

The screening of the electrostatic field by the counterions that accumulate near the charged plane can be represented by the effective thickness of the electrical double layer. In previous work [18] we have introduced the distance $x_{1 / 2}$, where the density of the number of the counterions (calculated relative to its value far from the charged plane) drops to half of its value at $x=0$ :

where $n_{\mathrm{ct}}(0)$ is density of the number of counterions at

Here, we generalize this condition so as to consider the finite dimension of the lattice by the distance of the closest approach of the ions $a / 2$ and require a drop of a fraction $\vartheta$ of the value of $n_{\mathrm{ct}}(a / 2)$ : 
$n_{\mathrm{ct}}\left(x_{\vartheta}\right)-n_{\mathrm{d}}=(1-\vartheta)\left(n_{\mathrm{ct}}(a / 2)-n_{\mathrm{d}}\right)$

We introduce another measure of the thickness of the electrical double layer, namely the distance $d_{\vartheta}$, defining the region that contains a certain fraction $\vartheta$ of the excess of the counterions

$\int_{a / 2}^{d_{\vartheta}}\left(n_{\mathrm{ct}}(x)-n_{\mathrm{d}}\right) \mathrm{d} x=\vartheta \int_{a / 2}^{d}\left(n_{\mathrm{ct}}(x)-n_{\mathrm{d}}\right) \mathrm{d} x$

For vanishing ion size (in the limit of the validity of the Poisson-Boltzmann theory) $x_{\vartheta}$ is expressed analytically:

$x_{\vartheta}=\frac{1}{\kappa} \ln \left(\frac{\left(\sqrt{1+(1-\vartheta)\left(\exp \left(\frac{-v_{\mathrm{ct}} e_{0} \Phi(0)}{k T}\right)-1\right)}+1\right)\left(1-\exp \left(\frac{v_{\mathrm{ct}} e_{0} \Phi(0)}{2 k T}\right)\right)}{\left(\sqrt{1+(1-\vartheta)\left(\exp \left(\frac{-v_{\mathrm{ct}} e_{0} \Phi(0)}{k T}\right)-1\right)}-1\right)\left(1+\exp \left(\frac{v_{\mathrm{ct}} e_{0} \Phi(0)}{2 k T}\right)\right)}\right)$

where

$\Phi(0)=-\frac{2 k T}{v_{\mathrm{ct}} e_{0}} \ln \left(\sqrt{1+(\sigma / c)^{2}}+|\sigma| / c\right)$

same size. Without this assumption the number densities of the ions could not be expressed analytically (as by Eqs. (2) and (3)). Therefore, in the model all particles have the same size. In previous work [18] we have arbitrarily adjusted the lattice constant to the size of solvent molecules, as we were interested in the general solution of the self-consistent expressions for the electrostatic potential, particle distribution functions and free energy. Here, we focus on the effect of the size of the particles; therefore, the choice of the lattice constant should be adjusted according to the relevant features under consideration. Far from the charged plane the electrostatic field does not depend on the lattice constant [18]. On the other hand, in the vicinity of the charged plane the counterions are accumulated while the coions are scarce. The influence of the counterions contained in the region close to the charged plane on the electrostatic field is the most important one [18]. Therefore, we propose that it is relevant to chose the lattice constant $a$ by the size of the counterions in the solution. Different solutions characterized by the kind of counterions can then be simulated by changing the lattice constant $a$ pertaining to the corresponding kind of counterions.

To study the effective thickness of the electrical double layer for different sizes of the counterions we solve Eq. (4), determine the distribution function given by Eq. (2) and calculate the parameters $x_{\vartheta}$ and $d_{\vartheta}$. The solution of Eq. (4) is obtained numerically by using the fourth-order Runge-Kutta method [21] starting far from the charged plane, and the integral in Eq. (10) is calculated using Simpson's method.

The ion density is defined only for $x>a / 2$, whereas the electrostatic field is defined for all $x \geq 0$ and is subject to the condition in Eq. (5). 


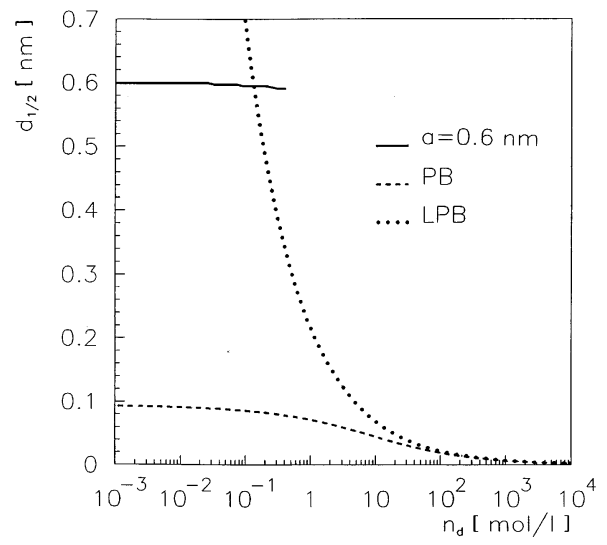

Fig. 2. The dependence of the thickness of the electrical double layer represented by the parameter $d_{1 / 2}$ on the bulk density of the number of the ions $n_{\mathrm{d}}$. The results of the nonlinearized Poisson-Boltzmann (PB) theory and the linearized PoissonBoltzmann (LPB) theory are also shown. The model parameters are $\varepsilon=78.5, T=310 \mathrm{~K}$, and $|\sigma|=0.4 \mathrm{~A} \mathrm{~s} / \mathrm{m}^{2}$.

\section{Results}

Fig. 1 shows the parameters $x_{9}$ (a) and $d_{9}$ (b) representing the effective thickness of the electrical doublelayer in dependence on the lattice size $a$ that represents the ion size. Three choices of $\vartheta$ are considered $(\vartheta=0.9$, 0.8 and 0.5 ). The points marked by the dots in Fig. 1(a) and (b) show the result of the Poisson-Boltzmann theory (Eqs. (15) and (11) respectively). The values of the parameters $d_{\vartheta}$ and $x_{\vartheta}$ are larger for higher $\vartheta$; however, qualitative dependence is equal for all three choices. For small values of $\vartheta$ both parameters diminish, whereas as $\vartheta$ approaches unity both the parameters

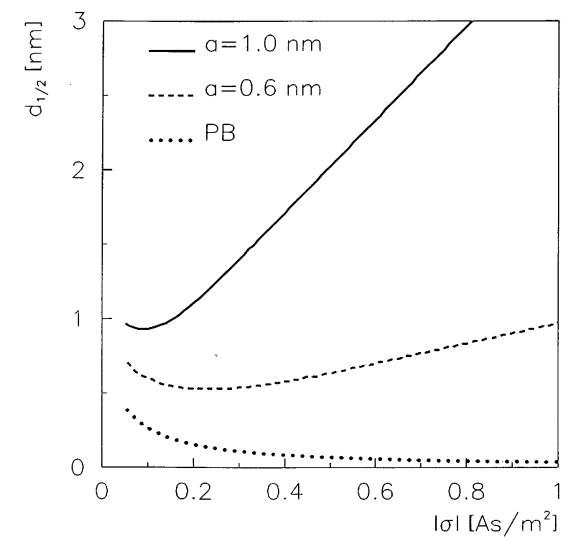

Fig. 3. The dependence of the thickness of the electrical double layer represented by the parameter $d_{1 / 2}$ on the surface charge density $|\sigma|$ of the $x=0$ plane for different lattice constants $a$. The result of the Poisson-Boltzmann (PB) theory is also shown. The model parameters are $\varepsilon=78.5$ and $T=310 \mathrm{~K}$, and $n_{\mathrm{d}}=0.1 \mathrm{~mol} / 1$. increase beyond every bound (not shown). It can be seen in Fig. 1 that the effective thickness of the electrical double layer increases with increasing size of the counterions, reaching values of several nanometers for lattice constants of about $1 \mathrm{~nm}$. The limit of small $a$ corresponds well with the Poisson-Boltzmann theory.

Fig. 2 shows the dependence of the parameter $d_{\vartheta}=1 / 2$ on the number density of the ions far from the charged plane $n_{\mathrm{d}}$ for a chosen lattice constant $a$. The curves calculated using the Poisson-Boltzmann theory and the linearized Poisson-Boltzmann theory are also shown. For small densities of the number of ions far from the charged plane $n_{\mathrm{d}}$ the effective thickness of the electrical double layer is constant in an interval of about three orders of magnitude. However, the value of the effective thickness is larger for larger lattice constants. The curve obtained by the Poisson-Boltzmann theory exhibits qualitatively the same behavior. In contrast, the linearized Poisson-Boltzmann theory yields a dependence that deviates qualitatively from the other results shown in Fig. 2. In this case, with decreasing $n_{\mathrm{d}}$, instead of reaching a plateau value, the effective thickness increases beyond every bound. Note that the curve corresponding to the finite size of counterions is defined only to a certain upper bound with respect to $n_{\mathrm{d}}$.

Fig. 3 shows the dependence of the parameter $d_{1 / 2}$, representing the effective thickness of the electrical double layer, on the surface charge density $|\sigma|$ of the $x=0$ plane. Two choices of lattice constant $a$ are given. If the surface charge density $|\sigma|$ increases, then the effective thickness of the electrical double layer containing counterions of a finite size decreases, reaches a minimum and then increases. The corresponding result of the Poisson-Boltzmann theory shows a continuous decrease of $d_{1 / 2}$ with increasing $|\sigma|$.

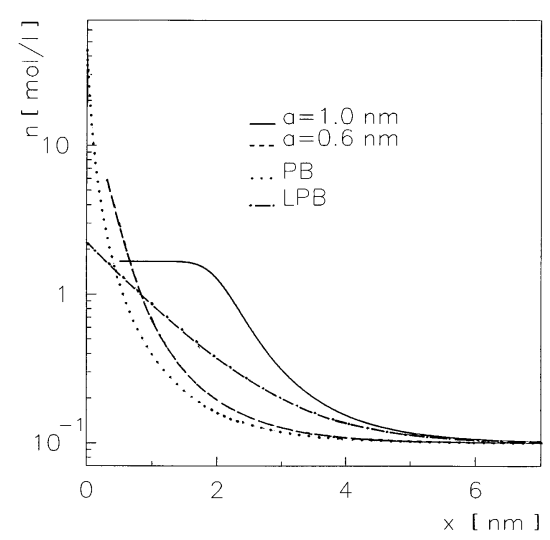

Fig. 4. The density profile of the counterions $n_{\mathrm{ct}}$ for different lattice constants $a$. The results of the nonlinearized PoissonBoltzmann (PB) theory and the linearized Poisson-Boltzmann (LPB) theory are also shown. The model parameters are $\varepsilon=78.5$ and $T=310 \mathrm{~K}, n_{\mathrm{d}}=0.1 \mathrm{~mol} / 1$, and $|\sigma|=0.4 \mathrm{~A} \mathrm{~s} / \mathrm{m}^{2}$. 


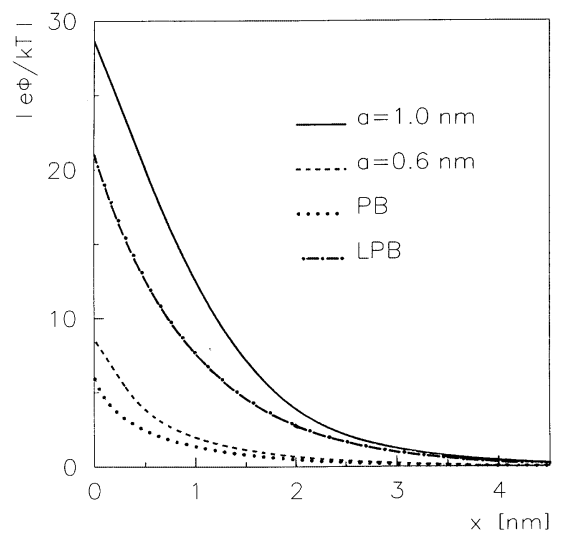

Fig. 5. The dependence of the electric potential on the distance from the charged plane $x$ for different lattice constants $a$. The results of the nonlinearized Poisson-Boltzmann (PB) theory and the linearized Poisson-Boltzmann (LPB) theory are also shown. The model parameters are $\varepsilon=78.5$ and $T=310 \mathrm{~K}$, $n_{\mathrm{d}}=0.1 \mathrm{~mol} / 1$, and $|\sigma|=0.4 \mathrm{~A} \mathrm{~s} / \mathrm{m}^{2}$.

Fig. 4 shows the profile of the density of the number of counterions, and Fig. 5 shows the electrostatic potential dependence on the distance from the charged plane. Two different lattice constants $a$ and the results of the Poisson-Boltzmann and the linearized PoissonBoltzmann theory are given. The density profile of the counterions in the vicinity of the charged plane depends strongly on the lattice constant, whereas far from the charged plane the density of the counterions is insensitive to the lattice constant. Note that the counterion density is defined only for $x>a / 2$. For large enough $a$ the profile exhibits a plateau region near the charged plane, whereas in the limit of vanishing $a$ the density of the number of the counterions $n_{\mathrm{ct}}$ converges towards the corresponding density obtained by the PoissonBoltzmann theory.

\section{Discussion}

We described the thickness of the electrical double layer by two characteristic lengths: (a) the distance where the excess of the counterions drops to a chosen fraction of the initial value $x_{\vartheta}$ and (b) the distance $d_{9}$ wherein a chosen fraction of all the excess counterions is contained. The values of $d_{\vartheta}$ are higher than the corresponding values of $x_{9}$. In the limit of vanishing ion size (at finite density of the number of the counterions far from the charged plane $n_{\mathrm{d}}$ and area density of charge of the charged plane $|\sigma|$ ) both parameters monotonously increase with increasing choice of $\vartheta$ from zero at $\vartheta=0$ and diverge as $\vartheta$ approaches unity. The parameter $\vartheta$ should, therefore, not be too low, as for small $\vartheta$ only a small part of excess counterions is involved in the region $x<d_{\vartheta}$. On the other hand, for $\vartheta$ very close to unity the thickness of the electrical double layer is almost independent of all the parameters, as the region must contain almost all the excess counterions. It is sensible to choose $\vartheta$ somewhere between 0.4 and 0.8 , where the effect of other different parameters is clearly exhibited.

Assuming close packing, the layer width can be approximated by $d_{\mathrm{cp}}=a^{3} e_{0} /|\sigma|$. This simple expression compares with the results shown in Fig. 1. For example, $d_{\mathrm{cp}}=2.5 \mathrm{~nm}$ for $a=1 \mathrm{~nm}$.

As the values of the effective thickness of the electrical double layer are of the same order as the lattice constant, the macroscopic quantities of the density of the number of ions and the dielectric constant are poorly defined. We have taken that the density of the number of particles is defined only for $x>a / 2, \varepsilon=78.5$ for $x>0$ and that the electrostatic field is $-\sigma / \varepsilon \varepsilon_{0}$ for $0<x \leq a / 2$.

In the limit of vanishing ion size (at finite bulk density of the number of counterions $n_{\mathrm{d}}$ ) and for very small area density of charge $|\sigma|$, both $d_{\vartheta}$ and $x_{\vartheta}$ approach the expression Eq. (16) by the Debye length.

In considering the effect of the bulk density of ions, a saturation in the thickness of the electrical double layer is obtained in a broad region over several orders of magnitude for a small bulk density of counterions $n_{\mathrm{d}}$ (Fig. 2). It can be emphasized that the linearized Poisson-Boltzmann theory may considerably overestimate the thickness of the electrical double layer even for moderate bulk counterion densities corresponding to the concentrations $10-100 \mathrm{mmol} / \mathrm{l}$ (Fig. 2). Moreover, the result of the linearized Poisson-Boltzmann theory deviates qualitatively from the corresponding result of the nonlinearized Poisson-Boltzmann theory, increasing beyond every bound as the bulk density decreases. In the limit of vanishing ion size, moderate $|\sigma|$ and vanishing bulk density $n_{\mathrm{d}}$, the parameter $x_{\vartheta}$ approaches the expression $x_{\vartheta}=A_{0} / 2 \pi l_{B}(1 / \sqrt{1-\vartheta}-1)$ and the parameter $d_{\vartheta}$ approaches the expression $d_{\vartheta}=A_{0} \vartheta / 2 \pi l_{B}(1-\vartheta)$, where $A_{0}$ is the effective area of the charged plane that bears one elementary charge and $l_{\mathrm{B}}=e_{0}^{2} / 4 \pi k T \varepsilon \varepsilon_{0}$ is the Bjerrum length. Note that both expressions are independent of $n_{\mathrm{d}}$.

Our results indicate that caution should be taken while using the Debye length in interpretation of the phenomena considering the electrical double layer. It has already been suggested that a description of the electrical double layer by the Debye length is oversimplified in diluted solutions where solution behavior can be conveniently studied by the electrolytic conductivity [11].

Considering finite size of ions qualitatively agrees with the nonlinearized Poisson-Boltzmann theory (Fig. 2). However, as the thickness of the electrical double layer is increased for larger counterions, the quantita- 
tive agreement between the result of the linearized Poisson-Boltzmann theory and the result obtained by considering finite size of ions may, for certain choices of $|\sigma|$ and $n_{\mathrm{d}}$, be better than the agreement between the result of the nonlinearized Poisson-Boltzmann theory and the result obtained by considering finite size of counterions. This is notable also in Figs. 4 and 5 .

It can be deduced from Figs. 1-4 that the screening of the electric field of the charged plane is less effective for larger ion sizes. Namely, in the case of larger lattice constant $a$, the electrostatic field protrudes further into the solution and the energy of electrostatic field $W^{\text {el }}$ is higher. The entropic contribution to the free energy reflects the rearranging of ions in the solution. In the case of larger ions the rearrangement of ions is extended further into the solution; however, the rearrangement of ions near the charged plane is much less pronounced.

The results regarding the counterion density profile (Fig. 4), which show a continuous decrease for small lattice constant and a plateau region for large lattice constants, reproduce the profiles obtained by Freise [14] (who introduced the excluded volume effect by the pressure-dependent chemical potential), by Wicke and Eigen [13,15] (who, in a thermodynamic approach, multiplied the density of the number of ions by a factor containing the number of the vacant sites), and by Borukhov et al. [19,20] (who used a systematic path integral approach within the lattice theory and a phenomenological approach based on the entropy of the solvent). It can be established that, for large counterions, two regions can be distinguished within the system: a saturated layer dominated by the steric repulsion and a diffuse layer extending into the solution.

Since the pioneering work of Gouy and Chapman, the description of the electrical double layer has been improved by considering molecular Hamiltonian models that take into account direct interactions between the charges in the system and solvent structure and interactions. Simulations were performed [7,22-28], and approximation approaches, such as the modified Poisson-Boltzmann theory [29,30], cluster expansion theory [31] and integral equation theories [32-34], have been developed.

However, these studies are comparatively demanding and are not convenient for everyday analysis of experimental data. Therefore, for such purposes the PoissonBoltzmann theory is still widely used [7]. Recently, theories have been developed that include some relevant property of the system, such as the excluded volume effect, in a relatively simple way $[13-15,18,19,35-37]$. Within the formalism used in this work, the effects of different sizes of counterion can be distinguished within a model that requires a solution of one relatively simple differential equation; therefore, it is suitable to apply in supporting the experiments. It is, however, important to estimate when the simplifications that are taken into account render the theory qualitatively insufficient to describe the phenomena of interest.

Liquid-state theory has shown that fluctuation terms are important [38]. Some models explicitly take into account the dipolar nature of the solvent $[36,39,40]$. Owing to the more detailed description, oscillations in the density of the ions near the charged plane occur. For monovalent ions in the solution the effects of the direct interactions of ions and of the solvent are negligible $[7,28,41]$. If the valence of the ions and the concentration of the ions are higher, direct interactions between the particles that constitute the system become more important.

In our model, we cannot account for the direct interactions owing to the limitations due to the mean field approximation. Also, the solvent is treated within the solvent primitive model [42]: on the one hand, as a set of particles that interact through the excluded volume effect and, on the other hand, as a medium characterized by a dielectric constant. We cannot obtain the oscillations of the ion density profiles. However, the calculated ion density profiles obtained by the Monte Carlo method [23] show that the amplitudes of the oscillations for univalent and divalent electrolytes are much smaller than the drop of the counterion density due to the screening of the electrostatic field of the charged plane. Although the results of the Monte Carlo simulations [7,23] are given for relatively low $\sigma$, where the effects of the direct interactions are relatively stronger, the amplitudes of the oscillations are so small that the oscillations would not affect the thickness of the electrical double layer as defined in our work.

The ion density profiles near the charged plane are, however, significantly influenced by direct interactions. We have made a quantitative comparison between the results of the Monte Carlo simulations and our results by comparing the parameter $x_{\vartheta}$. We have used the ion density profiles calculated by the Monte Carlo method [23], from which we could estimate the distance where the counterion density drops to $1-\vartheta$ of its value closest to the charged plane. For a univalent electrolyte, where $\sigma=0.0438 \mathrm{~A} \mathrm{~s} / \mathrm{m}^{2}$ and $n_{\mathrm{d}}=1 \mathrm{~mol} / 1$, the parameter $x_{4 / 5}$ obtained by the Monte Carlo method is indistinguishable from the corresponding $x_{4 / 5}$ obtained by our method, whereas the parameter $x_{1 / 2}$ differs by about $10 \%$. For a divalent electrolyte, where $\sigma=$ $0.0876 \mathrm{~A} \mathrm{~s} / \mathrm{m}^{2}$ and $n_{\mathrm{d}}=1.2 \mathrm{~mol} / 1$, the difference in $x_{4 / 5}$ is about $25 \%$ and about $45 \%$ in $x_{1 / 2}$. The ion diameter was $0.425 \mathrm{~nm}$ in both cases [23]. As this parameter used in the Monte Carlo calculations [7,23] describes rather small ions, the results obtained by our theory for the respective lattice constant are very close to the results of the Poisson-Boltzmann theory. Unfortunately, we found no data for large ions at high $\sigma$, where the effect of our model becomes important. In the future it would 
be of interest to perform a Monte Carlo simulation for such a system.

The evaluation of the different theoretical approaches can only be made by comparison of the theoretical results with experimental data. The experiments using neutron diffraction [43] and X-rays [8,9,44] show accumulation of the counterions in the vicinity of the charged plane and a rapid decrease of the density as the distance from the plane increases. Recently, experiments with large multivalent phosphotungstate ions in contact with a highly charged plane were performed $[8,9]$. A monolayer composed of eicosylamine $\left(\mathrm{C}_{20} \mathrm{H}_{43}-\mathrm{NH}_{2}\right)$ was formed at the electrolyte solution/ air contact. The solution contained phosphotungstate anions $\left(\mathrm{PW}_{12} \mathrm{O}_{40}^{3-}\right)$. The $\mathrm{pH}$ of the solution was low, so that the amine group facing the solution was fully ionized. Thus the headgroup plane was highly positively charged. The lateral pressure, and the area per eicosylamine molecule, and therefore the surface charge of the charged plane were varied by the compression barrier. The density of the number of the phosphotungstate counterions in the vicinity of the headgroup plane was measured by X-ray reflectivity and optical observations. It was found that most of the counterions are located within a single layer of monomolecular width. Fig. 6 shows the dependence of the number of the counterions close to the charged plane (per eicosylamine) on the effective area of the eicosylamine. The results of the measurements [9] for small areas per eicosylamine molecule (large $\sigma$ ) are compared with the corresponding theoretical results obtained by our theory and also by the Poisson-Boltzmann theory. The number of counterions close to the charged plane was calculated by integrating the counterion density within a layer of thickness $a$; it was considered that $a=1 \mathrm{~nm}$ [45]. It can be seen that by considering a finite size of counterions reproduces an increase in the number of

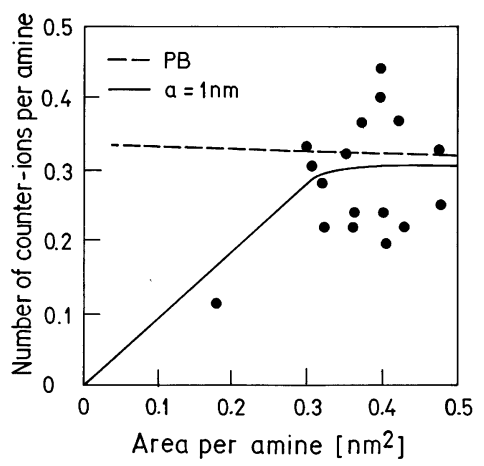

Fig. 6. The dependence of the counterions close to the charged plane per eicosylamine molecule on the effective area of the eicosylamine. The experimental data were taken from Ref. [9]. The values of the parameters used in calculations are $a=$ $1 \mathrm{~nm}, \varepsilon=78.5$ and $T=310 \mathrm{~K}$, and $n_{\mathrm{d}}=0.003 \mathrm{~mol} / 1$. counterions per eicosylamine with increasing area per eicosylamine (decreasing $\sigma$ ). This cannot be obtained by the Poisson-Boltzmann theory. However, in the above experiment, for a larger area per eicosylamine (small $\sigma$ ) it was found that more counterions approach the charged plane than are necessary to compensate the charge of the eicosylamine headgroups [8,9] (data not shown in Fig. 6). This effect can be described as an overcompensation of charge; however, it is also possible that small $\mathrm{H}_{3} \mathrm{O}$ coions that could not be detected by the experimental method used are present within the counterion-dense region [9].

We have failed to obtain precompensation of charge with our model where the charge is uniformly smeared over the charged plane by symmetric and nonsymmetric electrolytes of valences of coions and counterions up to four and also with symmetric electrolytes with added ions (coions and counterions). These results agree with the corresponding results obtained by a similar model [20]. This is due to the limitations of the model, probably due to the mean field description of the electrostatics. The overcompensation could be described within the primitive model by the molecular Hamiltonian theories, where other terms besides the mean field are taken into account [46]. However, it might also be possible that the discreteness of the charge could contribute to the explanation. Namely, it has been shown [47] that a multivalent polyion can cluster around a point charge of the opposite sign. Generalizing the origin of this effect, a counterion of a higher valence than the charge on the charged plane would approach the charge and reverse the sign of the complex if the complexes were sufficiently far apart.

The above experiments, where the density profiles were directly measured, did not confirm oscillations in density profiles that were predicted by the approaches based on the molecular Hamiltonian. The reported resolution of the experiments seems to be too low to yield a definite answer.

The features regarding the electrical double layer are of importance in amphiphilic layered systems, such as bilayer membranes. The contribution of the electrostatic forces to the free energy affects lateral pressure within the membrane [48] and influences the membrane curvature [49-52]. In the future it would be of interest to study the effect of ion size on the membrane curvature and, consequently, on the shape of aggregates made of amphiphilic molecules, such as phospholipid vesicles.

\section{Acknowledgements}

We thank V. Vlachy for valuable suggestions and discussions. 


\section{References}

[1] D. Andelman, in: R. Lipowsky, E. Sackmann (Eds.), Handbook of Biological Physics, vol. 1B, Elsevier, Amsterdam, 1995, p. 603.

[2] G. Barbero, L.R. Evangelista, N.V. Madhusudana, Eur. Phys. J. B 1 (1998) 327.

[3] R.J.F.L. Decarvalho, E. Trizac, J.P. Hansen, Phys. Rev. E 61 (2000) 1634.

[4] B.R. Horrocks, R.D. Armstrong, J. Phys. Chem. B 103 (1999) 11332.

[5] G. Gouy, J. Phys. Fr. 9 (1910) 457.

[6] D.L. Chapman, Philos. Mag. 25 (1913) 475.

[7] S.L. Carnie, G.M. Torrie, Adv. Chem. Phys. 56 (1984) 141.

[8] N. Cuvillier, M. Bonnier, F. Rondelez, D. Paranjape, M. Sastry, P. Ganguly, Prog. Colloid Polym. Sci. 105 (1997) 118.

[9] N. Cuvillier, F. Rondelez, Thin Solid Films 327-329 (1998) 19.

[10] G.V. Franks, S.B. Johnson, P.J. Scales, D.V. Boger, T.W. Healy, Langmuir 15 (1999) 4411.

[11] C. Wandrey, Langmuir 15 (1999) 4069.

[12] G. Lopez-Perez, D. Gonzales-Arjona, M. Molero, R. Andreu, Langmuir 15 (1999) 4892.

[13] E. Wicke, M. Eigen, Z. Elektrochem. 56 (1952) 551.

[14] V. Freise, Z. Elektrochem. 56 (1952) 822.

[15] M. Eigen, E. Wicke, J. Phys. Chem. 58 (1954) 702.

[16] V. Kralj-Iglič, A. Iglič, Electrotech. Rev. Slov. 61 (1994) 127.

[17] V. Kralj-Iglič, Elecrotech. Rev. Slov. 62 (1995) 104.

[18] V. Kralj-Iglič, A. Iglič, J. Phys. II Fr. 6 (1996) 477.

[19] I. Borukhov, D. Andelman, H. Orland, Phys. Rev. Lett. 79 (1997) 435.

[20] I. Borukhov, D. Andelman, H. Orland, Electrochim. Acta 46 (2000) 221.

[21] M. Abramowitz, I.A. Stegun, Handbook of Mathematical Functions, 10th edn, Dover, 1972, p. 897.

[22] W. van Megen, I. Snook, J. Chem. Phys. 73 (1980) 4656.

[23] I. Snook, W. van Megen, J. Chem. Phys. 75 (1981) 4104.

[24] G.M. Torrie, J.P. Valleau, J. Phys. Chem. 86 (1982) 3251.

[25] L. Guldbrand, B. Jönsson, H. Wenerström, P. Linse, J. Chem. Phys. 80 (1984) 2221.
[26] R. Kjellander, D.J. Mitchell, Chem. Phys. Lett. 200 (1992) 76.

[27] R. Kjellander, T. Åkesson, B. Jönsson, S. Marčelja, J. Chem. Phys. 97 (1992) 1424.

[28] R. Kjellander, Ber. Buns. Phys. Chem. 100 (1996) 894.

[29] S. Levine, G.M. Bell, J. Phys. Chem. 64 (1960) 1188.

[30] C.W. Outhwaite, L. Bhuiyan, J. Chem. Soc. Faraday II 79 (1983) 707.

[31] F.P. Buff, F.H. Stillinger, J. Chem. Phys. 39 (1963) 1911.

[32] L. Blum, J. Phys. Chem. 81 (1977) 136.

[33] D. Henderson, L. Blum, W.R. Smith, Chem. Phys. Lett. 63 (1979) 381.

[34] D. Bratko, V. Vlachy, Chem. Phys. Lett. 90 (1982) 434.

[35] R.D. Coalson, A.M. Walsh, A. Duncan, N. Ben-Tal, J. Chem. Phys. 102 (1995) 4584.

[36] T. Biben, J.P. Hansen, Y. Rosenfeld, Phys. Rev. E 57 (1998) R3727.

[37] L. Lue, N. Zoeller, D. Blankschtein, Langmuir 15 (1999) 3726.

[38] J.P. Hansen, I.R. McDonald, Theory of Simple Liquids, Academic Press, London, 1986.

[39] J.R. MacDonald, S.W. Kenkel, J. Chem. Phys. 80 (1984) 2168.

[40] S.W. Kenkel, J.R. MacDonald, J. Chem. Phys. 81 (1984) 3215.

[41] V. Vlachy, Annu. Rev. Phys. Chem. 50 (1999) 145.

[42] M. Lee, K.Y. Chan, D. Nicholson, S. Zara, Chem. Phys. Lett. 307 (1999) 89 and references cited therein.

[43] M.P. Hentschel, M. Mischel, R.C. Oberthür, G. Büldt, FEBS Lett. 193 (1985) 236.

[44] S. Kirchner, Biochim. Biophys. Acta 1279 (1996) 181.

[45] M.R. Spirlet, W.R. Busing, Acta Crystallogr. 154 (1987) 137.

[46] J.F. Joanny, Eur. Phys. J. B 9 (1999) 117.

[47] E. Gurovitch, P. Sens, Phys. Rev. Lett 82 (1999) 339.

[48] F. Jähnig, Biophys. Chem. 4 (1976) 308.

[49] I. Szleifer, D. Kramer, A. Ben-Shaul, W.M. Gelbart, S.A. Safran, J. Chem. Phys. 92 (1991) 6800.

[50] A. Ben-Shaul, in: R. Lipowsky, E. Sackmann (Eds.), Handbook of Biological Physics, vol. 1A, Elsevier, Amsterdam, 1995, p. 359.

[51] T. Chou, M.V. Jarić, E.D. Siggia, Biophys. J. 72 (1997) 2042.

[52] I.C. Carlsson, A. Fogden, H. Wennerström, Langmuir 15 (1999) 6150. 